\title{
Whole-genome sequencing of Mesorhizobium huakuii 7653R provides molecular insights into host specificity and symbiosis island dynamics
}

\author{
Shanming Wang ${ }^{1}$, Baohai Hao ${ }^{2}$, Jiarui $\mathrm{Li}^{3}$, Huilin Gu${ }^{1}$, Jieli Peng ${ }^{1}$, Fuli Xie ${ }^{1}$, Xinyin Zhao ${ }^{3}$, Christian Frech ${ }^{3}$,
} Nansheng Chen ${ }^{1,3^{*}+}$, Binguang $\mathrm{Ma}^{2^{*+}}$ and Youguo $\mathrm{Li}^{1^{*+}}$

\begin{abstract}
Background: Evidence based on genomic sequences is urgently needed to confirm the phylogenetic relationship between Mesorhizobium strain MAFF303099 and M. huakuii. To define underlying causes for the rather striking difference in host specificity between M. huakuii strain 7653R and MAFF303099, several probable determinants also require comparison at the genomic level. An improved understanding of mobile genetic elements that can be integrated into the main chromosomes of Mesorhizobium to form genomic islands would enrich our knowledge of how genome dynamics may contribute to Mesorhizobium evolution in general.

Results: In this study, we sequenced the complete genome of 7653R and compared it with five other Mesorhizobium genomes. Genomes of 7653R and MAFF303099 were found to share a large set of orthologs and, most importantly, a conserved chromosomal backbone and even larger perfectly conserved synteny blocks. We also identified candidate molecular differences responsible for the different host specificities of these two strains. Finally, we reconstructed an ancestral Mesorhizobium genomic island that has evolved into diverse forms in different Mesorhizobium species.

Conclusions: Our ortholog and synteny analyses firmly establish MAFF303099 as a strain of M. huakuii. Differences in nodulation factors and secretion systems T3SS, T4SS, and T6SS may be responsible for the unique host specificities of 7653R and MAFF303099 strains. The plasmids of 7653R may have arisen by excision of the original genomic island from the 7653R chromosome.
\end{abstract}

Keywords: Mesorhizobium huakuii 7653R, Genome sequencing, Comparative analysis, Host specificity, Symbiosis island

\section{Background}

Rhizobia are nitrogen-fixing soil bacteria constituting around 100 known species classified into 13 genera [1,2]. Mesorhizobium, whose growth rate is intermediate between that of genera Rhizobium and Bradyrhizobium, is one of the largest genera; it presently comprises 24 species found primarily in Asia, Europe, the Mediterranean

\footnotetext{
*Correspondence: chenn@sfu.ca; mbg@mail.hzau.edu.cn; youguoli@mail. hzau.edu.cn

${ }^{\dagger}$ Equal contributors

'State Key Laboratory of Agricultural Microbiology, Huazhong Agricultural University, Wuhan, Hubei, P. R. China

${ }^{2}$ Center for Bioinformatics, School of Life Science and Technology, Huazhong Agricultural University, Wuhan, Hubei, P. R. China

Full list of author information is available at the end of the article
}

region, and Africa [2,3]. Mesorhizobium huakuii and $M$. loti were two of the first species identified in the genus. The first known strain of $M$. huakuii was isolated from a winter-growing green manure crop, Astragalus sinicus, in Hubei, China in the 1940s by Huakui Chen [4], and was initially named Rhizobium huakuii by Wenxin Chen [5]. Rhizobium huakuii was later classified into Mesorhizobium gen. nov. and consequently renamed $M$. huakuii [6]. M. huakuii is a narrow-host-range rhizobium: it only induces indeterminate-type nitrogen-fixing nodules on the roots of $A$. sinicus, an economically important forage and green manure crop grown throughout eastern Asia in winter. The M. huakuii strain 7653R has been studied extensively and has been applied in sustainable agriculture 
for many years [7-9]. To facilitate comparative genomic investigation of the mechanism underlying this strain's symbiosis and its host-plant molecular interactions, the first specific aim of our research was to sequence, assemble, and annotate the entire genome of 7653R.

The first completely sequenced Mesorhizobium strain was $M$. huakuii bv. loti MAFF303099, initially considered a strain of $M$. loti [10]. Comparative sequence analysis of additional conserved genes (including $16 \mathrm{~S}$ rRNA, $g \ln A$, glnII, and $r e c A$ ) have suggested instead a closer phylogenetic relationship with strains of a different species, $M$. huakuii, prompting the hypothesis that MAFF303099 is a strain of M. huakuii [11]. Whole-genome sequencing of native $M$. loti strain R7A by the JGI GEBA project and various research findings related to R7A, such as genomic island mobility [12], the NifA-RpoN regulon and its symbiotic activation [13], and the role of the type-IV secretion system in genomic islands $[14,15]$, have provided a suitable reference strain and basis for the genomic comparison in this study. Consequently, our second goal was to determine whether genome-wide evidence supports the hypothesized assignment of MAFF303099 to M. huakuii.

Although MAFF303099 and 7653R may both be strains of the same species-M. huakuii, they display drastically different host preferences. Strain 7653R forms specific symbiosis with A. sinicus, whereas MAFF303099 forms determinant-type globular nodules and performs nitrogen fixing on several host plants of Lotus, including $L$. japonicus and L. corniculatus [16]. Our third aim was thus to identify genomic signatures possibly accounting for these differential host preferences.

Nodulation and nitrogen-fixation genes show remarkably different genomic locations in different genomes. While MAFF303099 and M. loti R7A have their nodulation and nitrogen-fixation genes concentrated in a long DNA region called a symbiosis island on their main chromosomes [12], the corresponding genes in 7653R are located primarily on plasmids [17]. Interestingly, nodulation and nitrogen-fixation gene locations of M. ciceri bv. biserrulae WSM1271 [18], M. australicum WSM2073 [19,20] and M. opportunistum WSM2075 [19,21], show patterns similar to those found in MAFF303099. These similarities suggest that genome recombination events and horizontal gene transfer are frequent in rhizobia. Our final objective was to define these genomic differences with the aim of elucidating their origin.

\section{Results and discussion}

\section{Complete sequencing of the $M$. huakuii 7653R genome}

Our 6,881,675-bp assembly of the $M$. huakuii 7653R genome consisted of three circular replicons of 6,364,365 bp (chromosome), 193,835 bp (plasmid pMhu7653Ra), and 323,475 bp (plasmid pMhu7653Rb) (Figure 1). The average
GC content of the whole genome was calculated to be 62.86\%, while plasmids pMhu7653Ra and pMhu7653Rb showed slightly lower GC levels (58.0\%). An overview of the GC content of the three replicons is shown on the 7653R genome physical maps (Figure 1). The main genome characteristics of 7653R and four other Mesorhizobium genomes (MAFF303099, WSM1271, WSM2075, and WSM2073) are summarized in Table 1. Although the five strains all belong to the same genus, they possess different numbers of plasmids: 7653R and MAFF303099 have two plasmids each, WSM1271 has only one, and WSM2073 and WSM2075 have none.

We predicted 7,205 protein-coding genes in the 7653R genome, a number essentially identical despite the different genome sizes to the number predicted in MAFF303099 (7,281 genes) [10]. 7653R was found to have the highest gene density among the five genomes, but have a lower ratio of genes with annotated functions, suggesting that it contains a higher ratio of genes with undefined functions. We examined the numbers and types of rRNAs and tRNAs of all five genomes predicted using the same strategy. We found that these five genomes had essentially identical numbers of rRNAs and tRNAs (Additional file 1: Table S1). However, the numbers of putative transposase genes predicted in these genomes were dramatically different (Table 1). As discussed later, this variation may have a profound differential impact on genome stability and horizontal gene transfer (HGT) events.

\section{Genomic evidence supporting MAF303099 as a strain of M. huakuii}

MAFF303099 has been hypothesized to be a strain of M. huakuii on the basis of comparative analysis of a few conserved genes in MAFF303099 and M. huakuii strains [11]. The availability of genome sequences of both strains has enabled us to re-examine their phylogenetic relationship.

\section{Genome-wide orthologs}

We identified a set of 7,414 orthologous groups among five Mesorhizobium genomes (7653R, MAFF303099, R7A, WSM1271, and WSM2073). Of these groups, 3,991 (54\%) were found to be conserved across all five genomes, with each group represented by at least one gene in each of the five genomes. We termed this subset of orthologous groups the core genome of Mesorhizobium. An additional 805 (11\% of orthologous groups) were observed to be present in four of the five genomes (Figure 2). The remaining orthologous groups $(28 \%$ or 2,104$)$ occurred in two or three genomes. Similar numbers and proportions of proteins predicted in $7653 \mathrm{R}(4,073 ; 57.5 \%)$ and MAFF303099 $(4,064 ; 57.1 \%)$ were present in the core genome, whereas $54.5 \%(4,064)$ of predicted proteins in R7A were present in the core genome. Among all pair- 


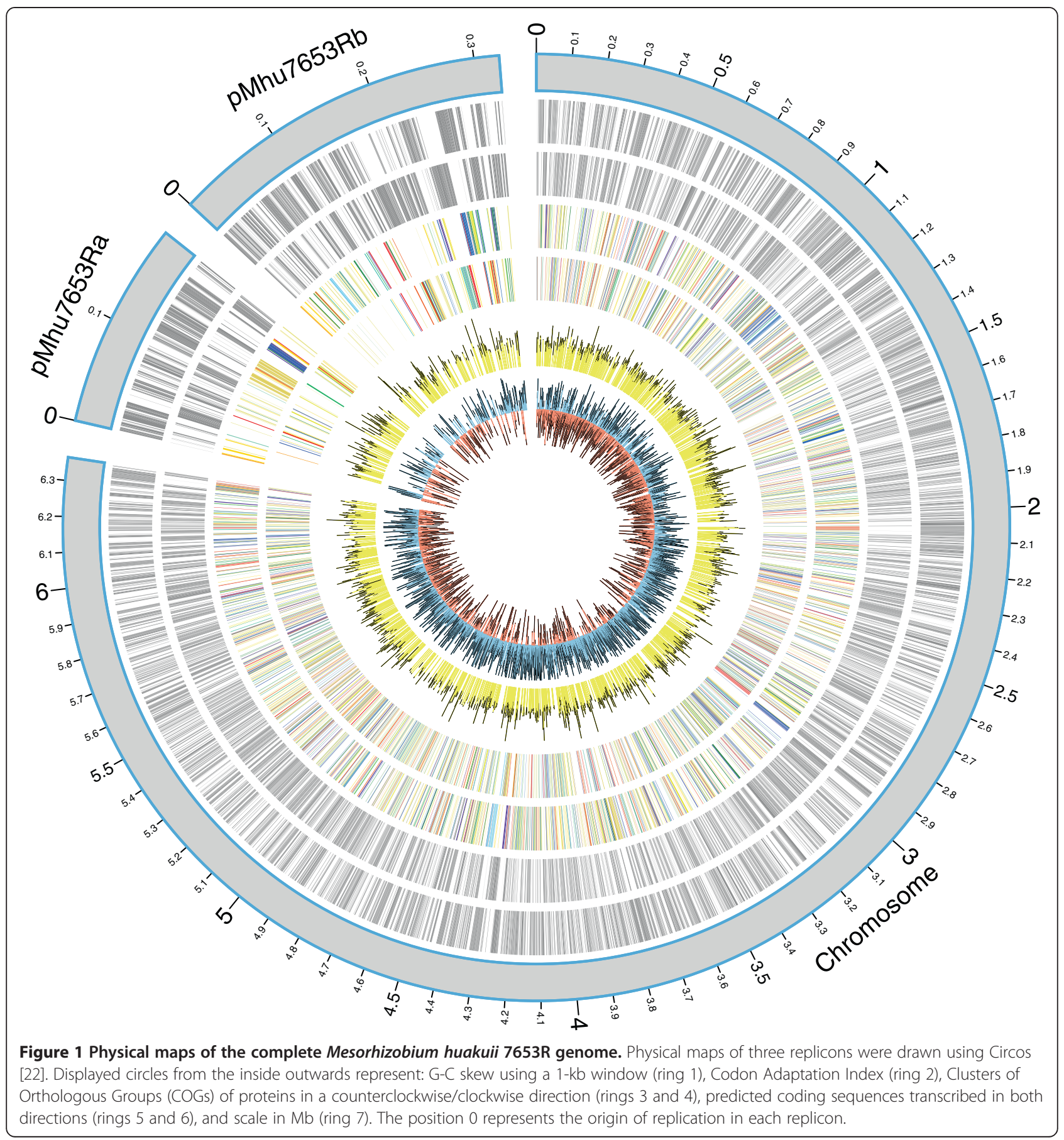

wise comparisons, the 7653R-MAFF303099 pair was found to share the most abundant orthologous proteins, followed by the R7A-MAFF303099 and WSM1271WSM2073 pairs; this ordering suggests that MAFF303099 has a closer phylogenetic relationship to 7653R than to R7A, WSM1271, and WSM2073. From the 4,073 7653R core genes, we randomly chose 210 single-copy genes (Additional file 2: Table S2) and performed hierarchical clustering analysis [23] based on their presence or absence in 16 representative rhizobial species. The clustering results also revealed a closer phylogenetic relationship between 7653R and MAFF303099 (Figure 3), further supporting MAFF303099 as a strain of M. huakuii.

\section{Synteny analysis}

The above analysis of orthologs suggested that MAFF 303099 is phylogenetically more closely related to $M$. huakuii strain $7653 \mathrm{R}$ than to strains of other species. We 
Table 1 General genomic features of Mesorhizobium huakuii 7653Rand four other mesorhizobial genomes

\begin{tabular}{|c|c|c|c|c|c|}
\hline & $7653 R$ & MAFF303099 & WSM1271 & WSM2705 & WSM2073 \\
\hline Size (bp) & $6,881,675$ & $7,596,297$ & $6,690,028$ & $6,884,444$ & $6,200,534$ \\
\hline $\mathrm{G}+\mathrm{C}$ content $(\%)$ & 62.86 & 62.51 & 62.56 & 62.87 & 62.84 \\
\hline Total no. of CDS & 7,205 & 7,281 & 6,264 & 6,508 & 5,792 \\
\hline CDS coverage $(\%)$ & 87.9 & 86.0 & 86.2 & 86.0 & 86.2 \\
\hline tRNA genes & 51 & 50 & 53 & 53 & 53 \\
\hline rRNA operons & 5 & 6 & 6 & 6 & 6 \\
\hline $\mathrm{Nb}(\%)$ CDS with assigned functions & $5,459(75.7)$ & $5,431(74.6)$ & $4,573(73.0)$ & $4,778(73.4)$ & $4,466(77.1)$ \\
\hline Average length of genes (bp) & 832 & 897 & 921 & 910 & 923 \\
\hline Putative transposases & 76 & 123 & 12 & 28 & 9 \\
\hline Tandem repeat sequences & 419 & 404 & 281 & 451 & 290 \\
\hline Transcriptional regulators & 285 & 330 & 407 & 474 & 376 \\
\hline Putative $A B C$ transporters-related proteins & 675 & 568 & 561 & 565 & 585 \\
\hline Putative two component systems-related proteins & 73 & 70 & 59 & 49 & 48 \\
\hline Putative sigma factor-related proteins & 29 & 24 & 30 & 32 & 28 \\
\hline
\end{tabular}

CDS: coding sequences.

Statistics for MAFF303099, WSM1271, WSM2075, and WSM2073 genomic features are based on NCBI annotations.

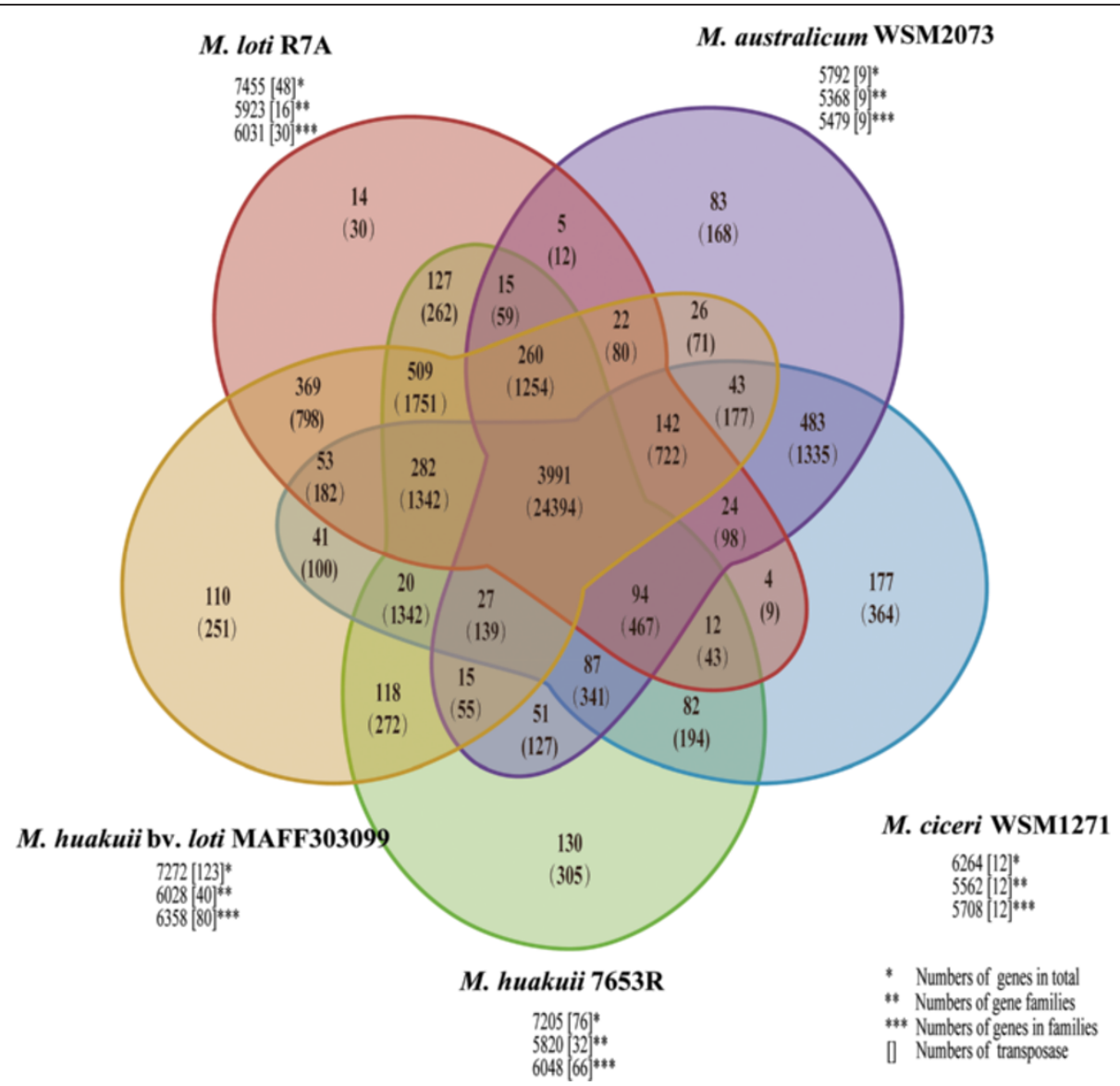

Figure 2 Core and accessory genome analyses of Mesorhizobium strains. The numbers of orthologous groups and related genes found in each intersection are shown. The numbers of genes found in related strains for each intersection are shown in parentheses. The numbers of transposase genes are shown in square brackets. Areas are not in scale. 


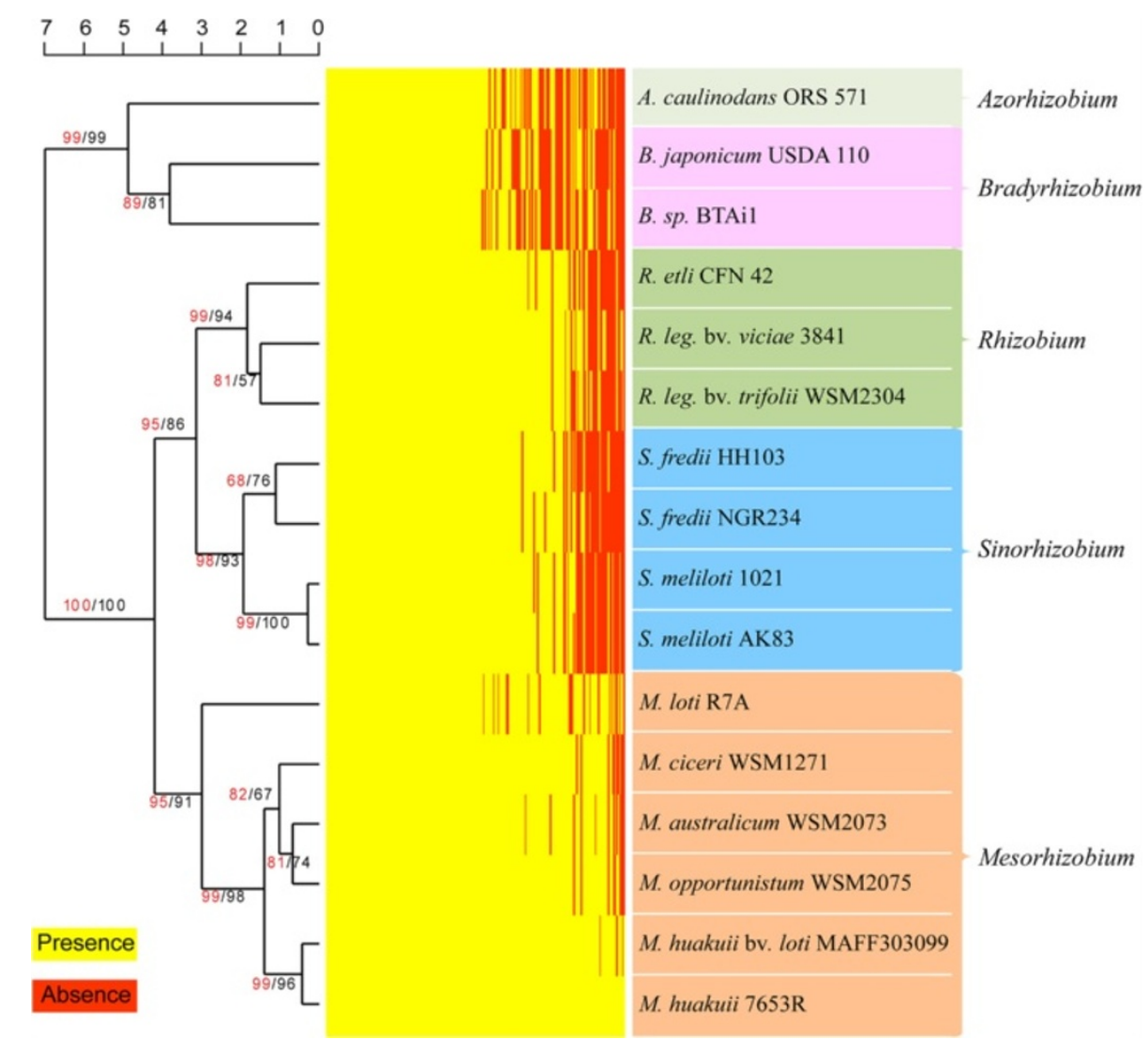

Figure 3 Hierarchical clustering analysis of rhizobia based on a heat map of $\mathbf{2 1 0}$ genes chosen from Mesorhizobium core genes. Gene homologs were chosen on the basis of BLASTP results ( $E$-value $\leq 1 \times 10^{-5}$; identity $\geq 35 \%$ ). Homolog presence and absence are indicated by yellow and red, respectively. The five genera for which multiple genomes were available are indicated in different colors. The numbers on the dendrogram represent bootstrap values.

further hypothesized that M. huakuii strain 7653R and MAFF303099 share larger synteny blocksbetween them than with the other three strains. To test this hypothesis, we carried out a synteny analysis of five strains (7653R, MAFF303099, WSM1271, WSM2073, and WSM2075) using a few complementary approaches. For convenience of comparison, we considered $d n a A$ to be the start position of the main chromosome and $\operatorname{rep} A B C$ to be the start position of plasmids in all five genomes. Mauve alignment [24] of chromosomes of the five strains revealed a remarkably consistent backbone (Figure 4A-D). Compared with WSM1271, WSM2073, and WSM2075, synteny blocks of the 7653R chromosome shared a longer average length and more consistent relative positions with MAFF303099. Additionally, fewer sequence inversions were observed between the chromosomes of 7653R and MAFF303099 than between 7653R and WSM1271, WSM2073, or WSM2075. We used OrthoCluster [25,26] to identify synteny blocks perfectly conserved between each pair of these five genomes, and assigned a score to each 7653R gene according to the length of the synteny (e.g., if gene $\mathrm{A}$ is in a synteny of seven genes, the score of gene A would be seven). We found that the mean score of all genes in synteny between 7653R and MAFF303099 was larger (10.46) than the mean scores between 7653R and WSM1271 (7.92), WSM2073 (8.24), or WSM2075 (8.86). Additionally, we performed a statistical test of significance, the results of which are shown Additional file 3: Table S3. Moreover, analyses of phylogenetic relationships based on the consistency of DNA sequences using Mauve and the Composition Vector using Cvtree [27] both indicated that 7653R has a closer relationship to MAFF303099 than to the other three Mesorhizobium strains (Figure 4E).

Thus, both ortholog and synteny analyses support a closer phylogenetic relationship between 7653R and MAFF303099 than with the other Mesorhizobium strains. These results provide further evidence that MAFF303099 is a strain of M. huakuii.

\section{Host specificity}

Although 7653R and MAFF303099 are both strains of the same species, M. huakuii, they display drastically different host specificities. While the strain 7653R forms a 


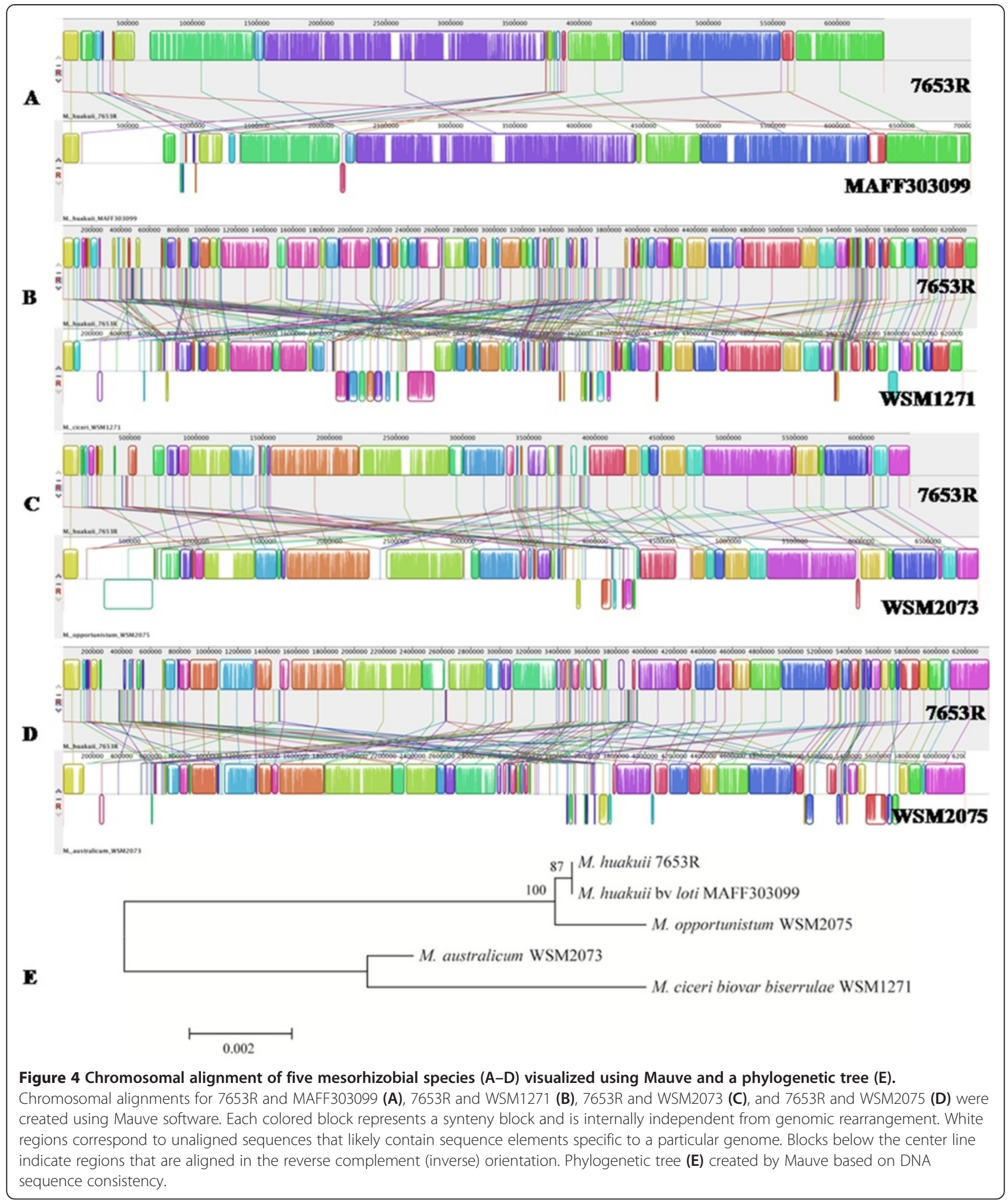

specific symbiosis with A. sinicus, MAFF303099 forms symbioses with several Lotus species host plants, including L. japonicus and $L$. corniculatus $[11,16]$. We aimed to determine what genomic features are responsible for such unique host preferences. Host specificity, an important trait underlying the interaction of rhizobia with their hosts, is still poorly understood [28]. Host switching or host jumping can often be traced to the modification 
of key microbial genes that facilitate the formation of particular host associations [29]. Because the determinants of host specificity of a bacterium mainly depend on three groups of signaling molecules-nodulation factors (NFs), surface polysaccharides, and secreted proteins [30], we explored genes that affect the biological synthesis of these signaling molecules in the genomes of these two strains and compared them with those of native $M$. loti strain R7A.

\section{Nodulation factors}

NFs, which are signaling molecules between symbiotic bacteria and plants, are produced by bacteria in response to flavonoids secreted by legume root hairs [31]. In most rhizobia, expression of nodulation genes (nod, nol, and noe) is needed for the biosynthesis and transport of NFs that induce nodule organogenesis. A total of 21 nodulation genes ( 2 nol genes and 19 nod genes) were identified in the 7653R chromosome and plasmids, while 33 nodulation genes were located in MAFF303099 (Figure 5). In contrast, in R7A, 24 nodulation genes were found to be present and all were found to be homologous with very high similarities to genes in MAFF303099. Comparison of nodulation genes between these three strains not only revealed some genes with high sequence similarity but also uncovered two striking differences likely related to NF synthesis.

First, genomic distribution of these nodulation genes was different between 7653R and MAFF303099. While all 33 nodulation genes in MAFF303099 were found on its chromosome, only 10 nodulation genes were present on the chromosome of 7653R, with 11 found on its plasmids ( 1 on pMhu7653Ra and 10 on pMhu7653Rb). Specifically, the 10 nod genes $(\operatorname{nod} A, B, C, D 2, E, F, G, H, I$, and $J)$ were identified in a $140-\mathrm{kb}$ genomic region of the pMhu $7653 \mathrm{Rb}$ plasmid of 7653R (Figure 6A). This genomic region also contained 6 fix genes (fix $A, B, C, L, U$, and $X$ ) and 12 nif genes (nifB, $D, E, H, K, N, Q, U, X, Z$, and two copies of nifA) (Figure 6B, C). The 10 nod genes were well conserved across all six genomes (Figure 5), as were the 6 fix genes and 12 nif genes (Additional file 1: Table S4). Although these nod genes individually exhibited strong conservation, major differences were observed in regard to their arrangement in the genomes. For example, the 10 nod genes on the pMhu7653Rb plasmid of 7653R were found to be segregated into two independent operons preceded by two nod-boxes (Figure 6A, C), with nod $A$ separated from $n o d B C$ by a $22-\mathrm{kb}$ genome region containing 35 genes [17]. In sharp contrast, orthologs of nod $A$ and $n o d B C$ in other Mesorhizobium strains, including MAFF303099 and R7A [10], are adjacent and localized on the same strand (Figure 6A).

Second, the numbers of nodulation genes putatively participating in NF synthesis were found to be different between these two strains. The nod gene nodH, required for NF synthesis in 7653R [32], had no ortholog in MAFF303099 and R7A (Figure 5). Each of the four nod genes in MAFF303099 $(\operatorname{nod} M, C, B$, and $A)$ had a substantially higher percentage identity (PID) with its ortholog in R7A than with its ortholog in 7653R (Figure 5). For example, nodC in MAFF303099 had a PID of $99 \%$ with its ortholog in R7A and a PID of $74 \%$ with its ortholog in 7653R (Figure 5). Interestingly, seven nodulation genes in MAFF303099 with orthologs in both 7653R and R7A were found to have substantially higher PIDs with their orthologs in R7A than with those in 7653R; nine nodulation genes in MAFF303099 had high PIDs with their orthologs in R7A but had no orthologs in 7653R. These results suggest that MAFF303099 may have obtained these 16 nodulation genes from an ancestor of R7A. Thus, although MAFF303099 shares 10 nodulation genes with high PIDs $(>92 \%)$ with $7653 \mathrm{R}$, it shares 24 nodulation genes with high PIDs with R7A. Furthermore, MAFF 303099 was found to have an additional five nodulation genes (nolT, $U, V, W$, and $X$ ). Taken together, 7653R and MAFF303099 have very different numbers of nodulation genes. Indeed, different nodulation genes are required for NF synthesis in these two strains. Of the 21 nod genes identified in 7653R, $12(\operatorname{nod} M, C, B, L, A, H, P, Q$, and two copies of nodE and $F$ ) are possibly key elements involved in the synthesis of the core NFs of 7653R [32] (Figure 7). In the M. loti strain R7A, nod genes organized in seven transcriptional units-noeKJ, nodZnoeLnolK, nodS, nodACIJnolO, nodB, nolL, and nodM-are needed for NF synthesis [12] (Additional file 4: Figure S1). Considering that many of the MAFF303099 nodulation genes showed higher PIDs with R7A genes, we further propose that nodulation genes required for the synthesis of NFs in MAFF303099 are closely related to those in R7A. This inference is consistent with a report that MAFF303099 and R7A may share the same steps of NF synthesis [32].

\section{Surface polysaccharides}

Rhizobial cell-surface polysaccharides, including cyclic- $\beta$ glucans (C $\beta G s)$, exopolysaccharides (EPSs), lipopolysaccharides (LPSs), and capsular polysaccharides (KPSs or K-antigens), are necessary for establishing successful symbiosis with their hosts to form effective root nodules [33]. Comparative genomics analysis revealed that the genes needed for the biosynthesis of C $\beta G$ s ( $n d v A$ and $n d v B$ ), EPSs (26 exo/exs genes; in Additional file 1: Table S5), and LPSs (Additional file 4: Figure S2 and Additional file 1: Table S6) are well conserved in all six genomes, suggesting that genes involved in the biosynthesis of surface polysaccharides are unlikely to contribute substantially to host preference differentiation. 


\section{Similarities (\%) between MAFF303099, WSM1271, WSM2075, and WSM2073 nodulation genes and those of $7653 R$}

\begin{tabular}{|c|c|c|c|c|c|c|c|}
\hline Genes & $7653 \mathrm{R}$ & MAFF303099 & $\begin{array}{l}\text { R7A (Similarity to } \\
\text { MAFF303099) }\end{array}$ & WSM1271 & WSM 2075 & WSM2073 & $\begin{array}{l}\text { Location } \\
\text { in 7653R }\end{array}$ \\
\hline nolA & MCHK_0037 & Mlr5623 (98) & - & Mesci_6005 (94) & Mesop_6654 (95) & Mesau_06048 (96) & $\mathrm{Chr}$ \\
\hline $\operatorname{nodP}$ & MCHK_1040 & Mr7575 (99) & - & Mesci_4953 (98) & Mesop_5464 (97) & Mesau_05015 (97) & $\mathrm{Chr}$. \\
\hline $\operatorname{nod} Q$ & MCHK_1041 & Mlr7576 (99) & - & Mesci_4952 (95) & Mesop_5463 (97) & Mesau_05014 (96) & $\mathrm{Chr}$ \\
\hline nodG & MCHK_1282 & $\mathrm{Mlr} 7850(97)$ & - & Mesci_4751 (96) & Mesop_5244(96) & Mesau_04805 (96) & $\mathrm{Chr}$. \\
\hline $\operatorname{nod} T$ & MCHK_2751 & M111107 (96) & - & Mesci_3479 (80) & Mesop_3967 (94) & Mesau_03744 (92) & Chr. \\
\hline nodL & MCHK_4332 & M112768 (95) & - & Mesci_2106 (89) & Mesop_2223 (90) & - & Chr. \\
\hline nolR & MCHK_4356 & $\mathrm{Mlr} 2794(92)$ & - & Mesci_2083 (76) & Mesop_2199(85) & Mesau_02118 (79) & $\mathrm{Chr}$. \\
\hline $\operatorname{nod} N$ & MCHK_4592 & Mlr3097 (99) & - & Mesci_1825 (99) & Mesop_1868 (99) & Mesau_01888 (99) & $\mathrm{Chr}$ \\
\hline $\operatorname{nod} F$ & MCHK_6006 & Mlr4951 (99) & - & Mesci_0525 (96) & Mesop_0540(96) & Mesau_00548 (96) & $\mathrm{Chr}$. \\
\hline nodE & MCHK_6007 & Mlr4953 (97) & - & Mesci_0524 (86) & Mesop_0539 (94) & Mesau_00549(97) & $\mathrm{Chr}$ \\
\hline nodH & MCHK_ 8281 & - & - & - & - & Mesau_05914(80) & $\mathrm{pMHb}$ \\
\hline$n o d F$ & MCHK_8323 & Mlr5821 (56) & - & Mesci_5857 (82) & Mesop_6431 (82) & Mesau_05904 (82) & $\mathrm{pMHb}$ \\
\hline $\operatorname{nod} E$ & MCHK_8324 & Mlr5822 (72) & - & Mesci_5858 (86) & Mesop_6432(86) & Mesau_05905 (86) & $\mathrm{pMHb}$ \\
\hline $\operatorname{nod} G$ & MCHK_8325 & - & - & Mesci_5859 (93) & Mesop_6433 (93) & Mesau_05906 (93) & $\mathrm{pMHb}$ \\
\hline nodJ & MCHK_ 8282 & Mlr6166 (74) & Msil26 (99) & Mesci_5866 (88) & Mesop_6440(88) & Mesau_05913 (89) & $\mathrm{pMHb}$ \\
\hline nodl & MCHK_8283 & Mlr6164 (78) & Msil27 (99) & Mesci_5865 (89) & Mesop_6439 (89) & Mesau_05912(90) & $\mathrm{pMHb}$ \\
\hline $\operatorname{nod} C$ & MCHK_8284 & Mlr6163 (73) & Msil28 (97) & Mesci_5864 (81) & Mesop_6438 (81) & Mesau_05911 (81) & $\mathrm{pMHb}$ \\
\hline $\operatorname{nod} B$ & MCHK_8285 & $\operatorname{Mlr} 6175(66)$ & Msi122 (99) & Mesci_5863 (79) & Mesop_6437 (78) & Mesau_05910(78) & $\mathrm{pMHb}$ \\
\hline $\operatorname{nod} A$ & MCHK_8321 & Mlr8755 (67) & Msil29 (92) & Mesci_5856 (84) & Mesop_6430(84) & Mesau_05903 (84) & $\mathrm{pMHb}$ \\
\hline $\operatorname{nodM}$ & MCHK_7093 & $\mathrm{Mlr} 6774(70)$ & Msi038 (99) & Mesci_4136 (84) & Mesop_4114 (84) & Mesau_03875(84) & $\mathrm{pMHa}$ \\
\hline nodD2 & MCHK_8286 & M116179 (78) & Msil18 (98) & Mesci_5861 (85) & Mesop_6435 (85) & Mesau_05908 (85) & $\mathrm{pMHb}$ \\
\hline $\operatorname{nodDl}$ & - & Mlr6182 & Msil15 (99) & Mesci_5855 & Mesop_6429 & Mesau_05902 & \\
\hline nolL & - & M1r8757 & Msil16 (98) & - & - & - & \\
\hline nolo & - & Mlr6171 & Msil25 (99) & Mesci_5868 & Mesop_6442 & Mesau_05915 & \\
\hline nolK & - & Mlr8749 & Msi353 (99) & - & - & - & \\
\hline noel & - & Mlr5849 & Msi354 (99) & - & - & - & \\
\hline noed & - & $\mathrm{Mlr} 5802$ & Msi383 (99) & - & - & Mesau_05022 & \\
\hline noeK & - & $\mathrm{M} \operatorname{lr} 5801$ & Msi384 (99) & - & - & Mesau_05021 & \\
\hline nods & - & Mlr6161 & Msil31 (98) & - & - & - & \\
\hline nodZ & - & Mlr5848 & Msi355 (98) & - & - & - & \\
\hline nodW & - & - & - & - & Mesop_5572 & - & \\
\hline nodU & - & - & - & Mesci_5894 & Mesop_6463 & Mesau_05938 & \\
\hline nolX & - & M116337 & - & - & - & - & \\
\hline nolW & - & M116338 & - & - & - & - & \\
\hline nolT & - & Mlr6339 & - & - & - & - & \\
\hline nolU & - & Mlr8764 & - & - & - & - & \\
\hline nolV & - & Mlr6341 & - & - & - & - & \\
\hline
\end{tabular}

Figure 5 Similarities (\%) between MAFF303099, WSM1271, WSM2075, and WSM2073 nodulation genes and those of 7653 R.

\section{Secretion system}

Proteins secreted by some rhizobial strains play an important role in infection of leguminous plant roots and establishment of a mutually beneficial symbiosis. Different types and numbers of protein secretion systems are present in almost all rhizobial strains. By means of similarity searches using protein secretion genes identified in other Gram-negative bacteria as queries, we identified 101 genes related to secretory processes in the 7653R genome. These genes and proteins are involved in 12 putative protein secretion systems: a general export pathway, four separate type-I systems, a twin-arginine translocase secretion system, one functional type-III system (T3SS), two type-IV systems (T4SSs), one type-V 

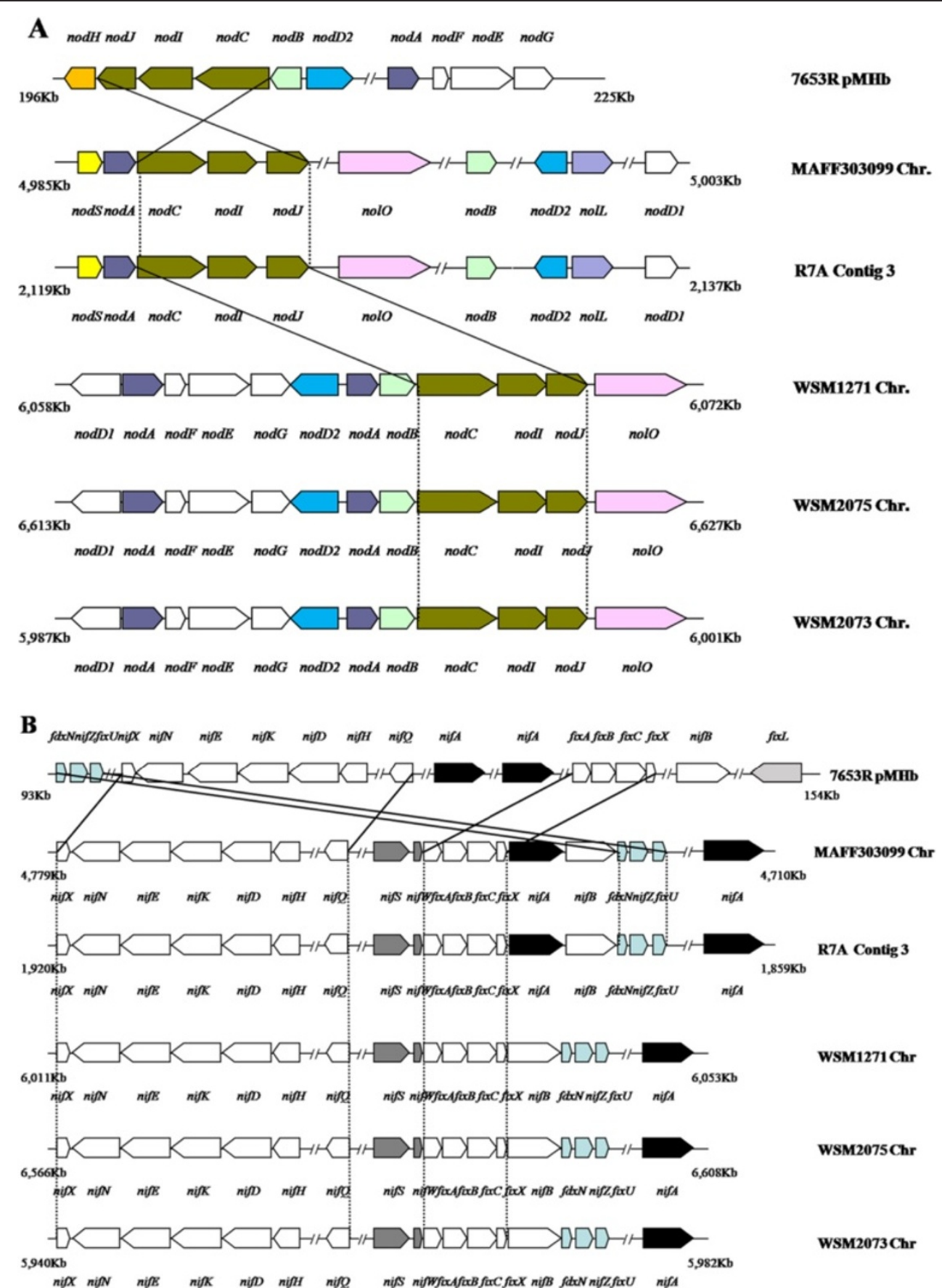

WSM2075Chr

WSM2073Chr

C

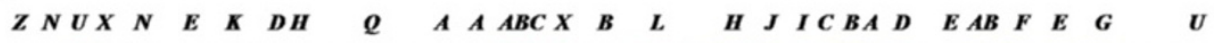

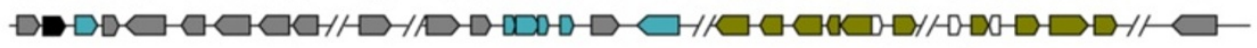
$\begin{array}{lllll}93 \mathrm{~Kb} & \text { T } & & 154 \mathrm{~Kb} & 244 \mathrm{~Kb}\end{array}$

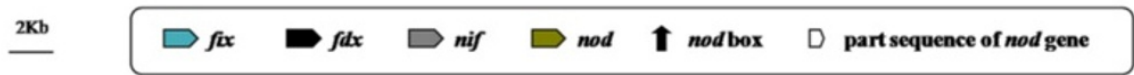


(See figure on previous page.)

Figure 6 Arrangement of nodulation genes (A), nitrogen fixation genes (B), and gene clusters in the 7653R plasmid pMhu7653Rb (C). Double slash marks represent genome regions that are not shown. In the clusters, nitrogen-fixation genes (B) conserved among the six strains are represented by white arrows, while those varying in copy number, location, or transcriptional orientation are shown in different colors. Nodulation and nitrogen-fixation gene clusters $(\mathbf{C})$ in 7653R plasmid pMhu7653Rb. Genes are colored according to their names. Double slash marks represent genome regions that are not shown. Arrows indicate the location of potential nod-boxes.

autotransporter, and two putative type-VI secretion systems (T6SSs) (Table 2).

Our comparative analysis of these secretion systems in the genomes of the two M. huakuii strains revealed important differences in three secretion systems: T3SS, T4SS, and T6SS. Gene clusters encoding the major and conserved components of T3SSs are present in diverse and distantly related rhizobia $[34,35]$. The $7653 \mathrm{R}$ genome was found to contain a complete T3SS on the pMhu 7653Rb plasmid, with gene organization conserved with respect to MAFF303099. Proteins secreted by rhizobial T3SS are called nodulation outer proteins (Nops) and can be divided into two types: effectors and helper proteins. T3SSs of both 7653R and MAFF303099 have three helper proteins, NopA, NopB, and NopX, but different candidate effectors: NopP in 7653R and NopC in MAFF303099 (Additional file 5: Table S7). Although T3SS and its secreted effectors are dispensable for rhizobial infection and nodulation, they may function as facilitators superimposed on the Nod-factor signaling pathway and modulate host range in a genotype-specific manner [28]. Thus, T3SS might be one determinant of host range variation in 7653R and MAFF303099. The Vir system, an important example of a T4SS, is usually formed by 12 proteins, VirB1-VirB11 and VirD4. Except for VirB1 and VirB7, these proteins are encoded by genes on plasmid pMhu 7653Ra. Interestingly, neither VirB1 nor VirB7 are present in MAFF303099 and R7A [14]. The Vir systems of 7653R and MAFF303099 are thus essentially identical. In contrast, the T4SS Trb system was found to differ between 7653R and the other five Mesorhizobium strains; in particular, 7653R has no trb gene, whereas MAFF303099 has 19 trb genes (Table 2). The T6SS apparatus is assembled by a conserved set of proteins whose functions are closely related to bacterial pathogenesis and host cell survival [36]. Two T6SSs were found in the 7653R genome, while one each was identified in MAFF303099 and R7A genomes (Table 2).

Taken together, our analysis revealed that the two $M$. huakuii strains 7653R and MAFF303099 have substantial differences in the number and arrangement of genes responsible for synthesizing NFs, and also differ with respect to secretion systems T3SS, T4SS, and T6SS. These differences may contribute to the establishment of differential host specificity.

Changes in host specificity determinants-for example, by acquisition of new genetic elements that grant a selective advantage in a particular host environmentcan have a great impact on host range and may lead to host jumps [29]. Both intrageneric and intergeneric HGT have been reported as important mechanisms for the spread of symbiotic capacity in the Salado River Basin [16]. Intrageneric HGT might be a main pathway to change symbiotic capacity in MAFF303099. Mesorhizobium strains isolated from A. sinicus in Japan, designated as $M$. huakuii subsp. rengei, are able to coexist with $M$. loti strains and thus have a chance to exchange genetic information through conjugation. The ancestral strain of $M$. huakuii presumably derived some genetic information from native $M$. loti strains, thereby introducing genetic variation in host specificity determination. The ancestral strain eventually evolved into strain MAFF303099, which can form an effective symbiotic relationship with Lotus corniculatus. The introduction of novel genetic variation by HGT is typically accompanied by the acquisition and incorporation of genetic fragments or intact transcriptional units into the genome [37]. Although NFs and secreted effectors of T3SS in MAFF303099 are associated with genetic fragments and intact transcriptional units, we still cannot confirm the underlying causes of the host specificity changes: there may be a continuum that ranges from changes in single residues to gene domains, whole genes, and eventually entire genomic islands (GEIs) [29]. Consequently, much remains to be learned about whether many or only a few gene loci are involved in the determination of nodulation specificity. Moreover, genes from leguminous plants, such as the $R$-gene from soybean [28], can also participate in the control of genotype-specific infection and nodulation.

\section{Symbiosis island dynamics and the origin of symbiotic plasmids}

Although the chromosomes of 7653R and MAFF303099 showed good overall co-linearity, a large, approximately $610-k b$ genomic region unique to MAFF303099 was identified (Figure 4A). Comparison of 7653R genomic structures to genomes of R7A and MAFF303099 using the ACT (Artemis comparison tool) [38] confirmed this observation (Additional file 4: Figure S3). Such genomespecific sequences were also noticed in similar positions in the other three genomes (WSM1271, WSM2075, and WSM2073) (Figure 4B-D), which was verified through genome alignment using PROmer (PROtein MUMmer) 


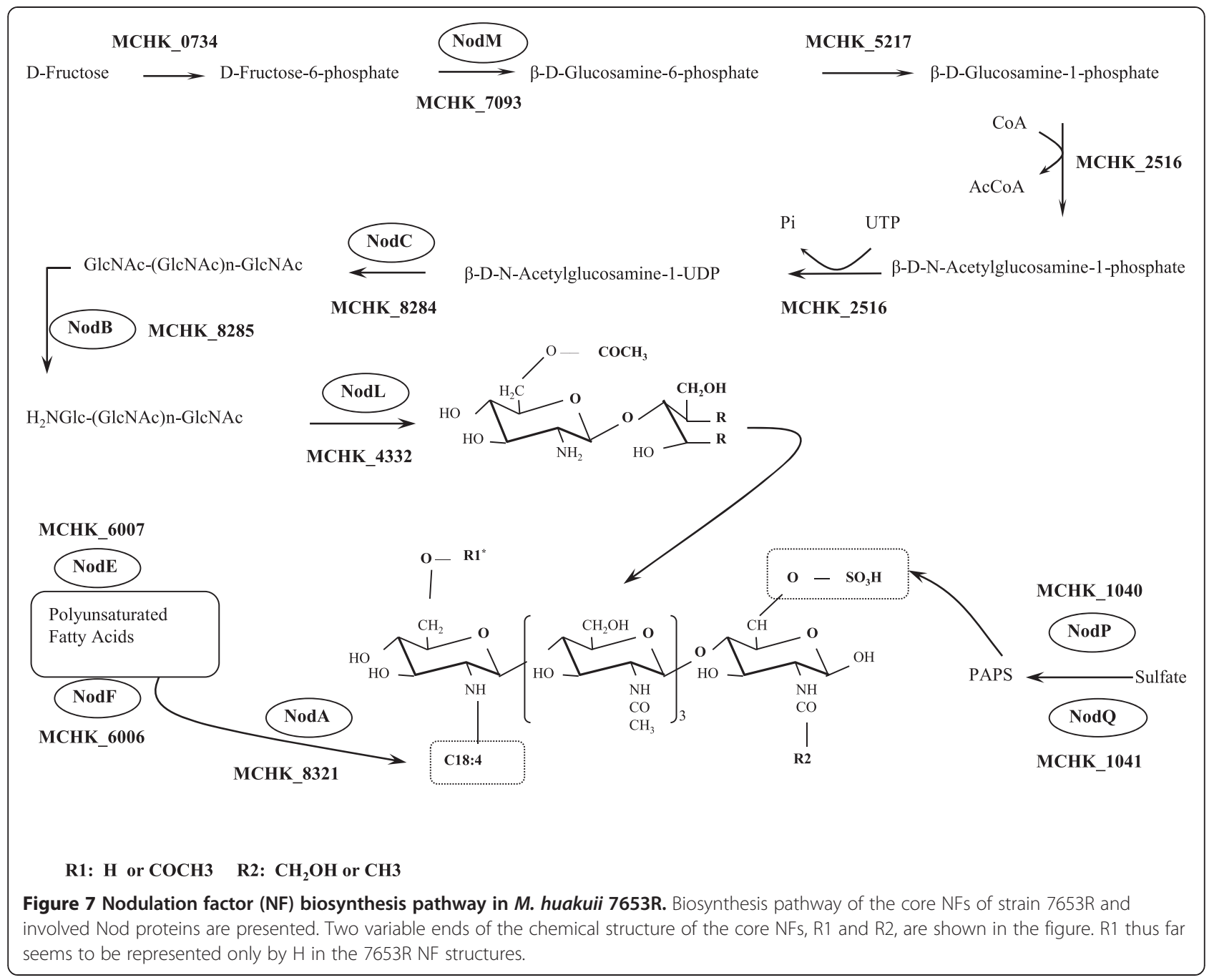

[39] (Figure 8). These genome-specific regions harbor most nodulation and nitrogen-fixation genes. Interestingly, homologs of these nodulation and nitrogenfixation genes can be traced to the two 7653R plasmids, suggesting a connection between the 'missing' DNA fragment and these symbiotic plasmids (Figure 8).

Of the five Mesorhizobium strains whose genomes have been completely sequenced (excluding R7A with its incomplete genome data), only $7653 \mathrm{R}$ has symbiotic plasmids. In contrast, all other strains either have no plasmids, or their plasmids do not contain genes involved in symbiosis. Thus, while the nodulation and nitrogenfixation genes are localized on the plasmids as a symbiosis island in 7653R, they are localized on the main chromosomes of the other four strains. Global genome alignment between 7653R and the other genomes revealed that the symbiosis islands are positioned in a synteny gap region that corresponds to the genome-specific region in MAFF303099 and the gap in 7653R (Figure 8 and Additional file 4: Figure S3), suggesting that the plasmids were excised from the main 7653R chromosome. Plasmids of $7653 \mathrm{R}$ and these genome-specific regions found in the other four genomes are thus likely GEIs. To test this hypothesis, we examined these genome-specific regions i.e., symbiosis islands, using IslandViewer, a program for finding GEIs [40]. As expected, IslandViewer identified these MAFF303099, WSM1271, WSM2073, and WSM2075 symbiosis islands as typical GEIs (Additional file 4: Figure S4). These predictions are supported by the results of further analysis of genomic features. First, plasmids of 7653R and the other four GEIs have similar sizes (514-611 kb) and similar GC content (58-59\%), which is strikingly lower than that of the corresponding genome (62.51-62.87\%). Second, codon usage of 7653R plasmid ORFs is significantly different from that of the chromosome but surprisingly consistent with those of the other four GEIs (Additional file 4: Figure S5). Third, T3SSs and/ or T4SSs of the five strains are all located in the corresponding candidate GEIs. Fourth, a highly conserved tRNA (Gly) gene is found in the vicinity of the candidate GEI in 
Table 2 Numbers and distributions of genes associated with different types of secretion systems in mesorhizobial genomes

\begin{tabular}{|c|c|c|c|c|c|c|}
\hline \multirow[t]{2}{*}{ Secretion system and characteristics } & \multicolumn{6}{|c|}{ Gene numbers associated with the formation of different types of secretion systems } \\
\hline & $7653 R$ & MAFF303099 & R7A & WSM1271 & WSM2075 & WSM2073 \\
\hline \multicolumn{7}{|l|}{ Type I } \\
\hline AprD & 4 & 3 & 2 & 2 & 2 & 3 \\
\hline AprE & 4 & 3 & 1 & 2 & 2 & 3 \\
\hline TolC & 2 & 1 & 1 & 2 & 2 & 2 \\
\hline \multicolumn{7}{|l|}{ Type II } \\
\hline GSP (general secretion pathway) & & 11 & 8 & 11 & & \\
\hline Sec pathway & 7 & 7 & 7 & 7 & 7 & 7 \\
\hline \multicolumn{7}{|l|}{ Type III } \\
\hline Rhc pili & 22 & 22 & & & & \\
\hline \multicolumn{7}{|l|}{ Type IV } \\
\hline F-type (Vir) & 10 & 10 & 9 & 12 & 12 & 11 \\
\hline F-type (Trb) & & 19 & 10 & 7 & 9 & 16 \\
\hline P-type (Flp and attachment) & 16 & 16 & 15 & 16 & 16 & 16 \\
\hline \multicolumn{7}{|l|}{ Type V } \\
\hline Autotransporter & 1 & 1 & 1 & 1 & 1 & 1 \\
\hline Type VI & 26 & 18 & 18 & 18 & & \\
\hline TAT (twin arginine) & 3 & 3 & 3 & 3 & 3 & 3 \\
\hline SRP & 5 & 5 & 5 & 5 & 5 & 5 \\
\hline Mechanosensitive channels & 1 & 1 & 1 & 1 & 1 & 1 \\
\hline Total & 101 & 120 & 81 & 87 & 60 & 68 \\
\hline
\end{tabular}

all five Mesorhizobium strains except for 7653R. In 7653R, plasmids possess the same characteristics as the other four GEIs located in specific genome regions. We propose that the plasmids of 7653R were formed during evolution by the excision of the GEI from the 7653R chromosome, as described previously in other systems [41].

Because the five GEIs likely share a common ancestor, we expected them to maintain well-conserved syntenic relationships. Although the GEIs in WSM1271, WSM2075, and WSM2073 displayed conserved synteny, the GEIs in these three strains and two other strains surprisingly showed little resemblance in regard to gene organization. We noticed that $80 \%$ of all transposase genes in the entire $7653 \mathrm{R}$ genome are concentrated on its plasmids. This enrichment of transposase genes on the plasmids of $7653 \mathrm{R}$ resembles that of the MAFF303099 GEI, which possesses 89 predicted transposase genes-86\% of all transposase genes in the entire MAFF303099 genome. Similarly, 85\% (41) of all transposase genes identified in the entire contigs of R7A are found in the symbiosis island of contig 3. In contrast, the GEIs of the other three Mesorhizobium strains harbor only a few transposase genes, and they show highly conserved synteny. On the basis of this observation, we propose that the enrichment of transposase genes in the GEIs of 7653R and MAFF303099 caused a disruption in gene order within their GEIs, whereas the lack of transposase genes in the other three Mesorhizobium strains helped to maintain their GEI synteny. The question then arises: what is the source of these transposase genes in the GEIs of 7653R and MAFF303099? One likely source is HGT. Previous analysis of nodulation genes has proved that the GEI of MAFF303099 has acquired many foreign genes by HGT [42]. Our clustering analysis of transposase genes in the plasmids of 7653R and the MAFF303099 GEI revealed that most of them belong to different families, suggesting that these transposase genes were likely acquired via HGT. Thus, these five Mesorhizobium strains may have inherited their GEIs from a common ancestral GEI, which later underwent various degrees of change.

It has been speculated that GEIs may be derived from integrating mobile genetic elements such as plasmids or phages. Their acquisition by HGT and integration with the host chromosome by site-specific recombination might lead to the formation of a new GEI [37]. Slater et al. have proposed that integration of an ancestral intragenomic translocation recipient (ITR) plasmid into the main chromosome is an important evolutionary pathway in Rhizobiales [43]. Bradyrhizobium and Mesorhizobium strains with a few or no relatively small plasmids are 


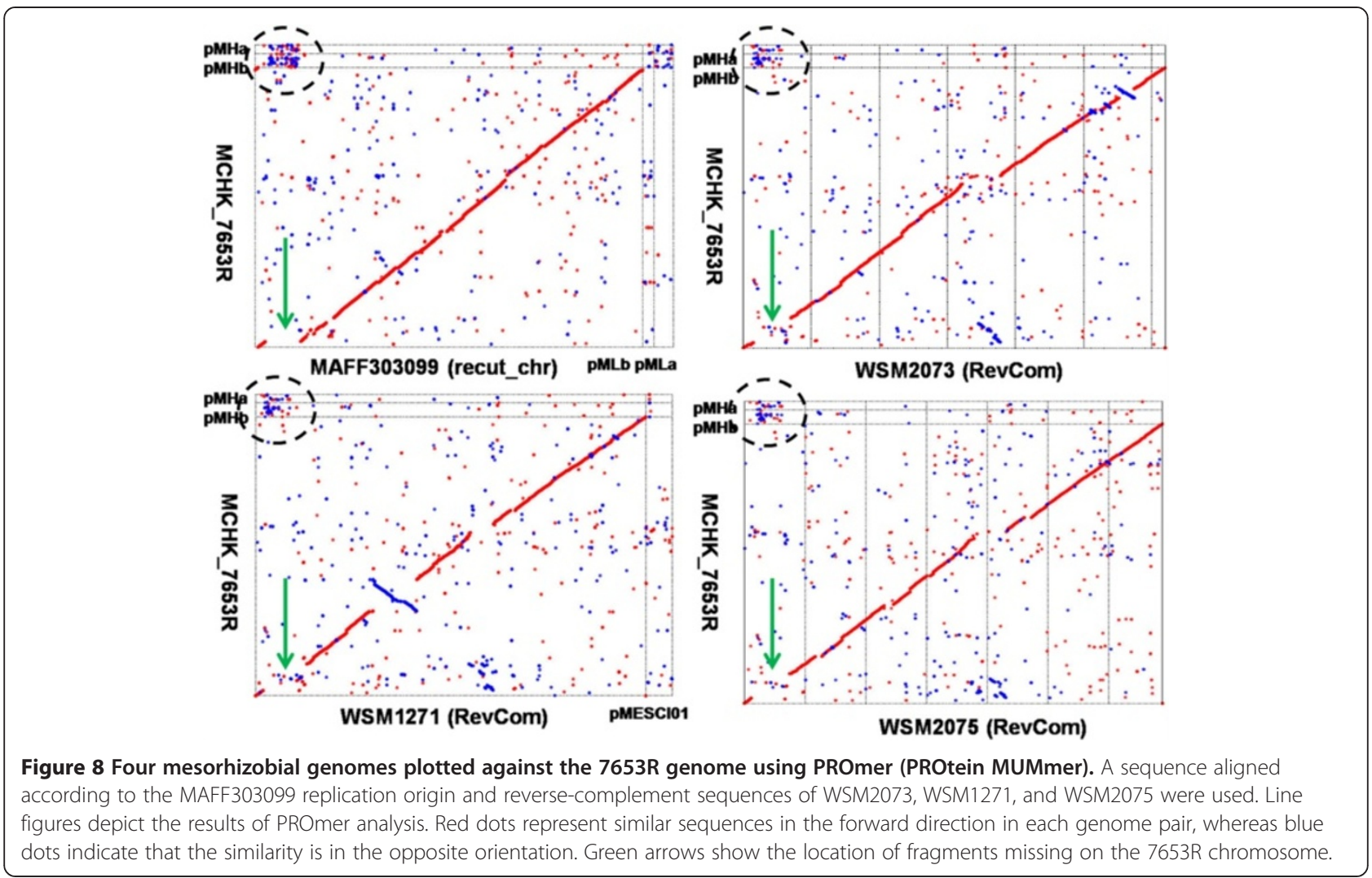

typically cited as examples, although the sole evidence for this viewpoint is the presence of ITR plasmid gene clusters and other plasmid genes on the chromosomes of these species. Aside from plasmid genes shown on chromosomes, further evidence based on genomic structure, nucleotide composition, and transposase genes was used in this study to infer a possible evolutionary pathway explaining GEI formation (Figure 9). In our proposed scenario, integration of an ITR plasmid into an ancestral Mesorhizobium main chromosome would be followed by the formation of a new GEI-the original parent of the present-day chromosomal GEIs of the four fully sequenced Mesorhizobium strains. Because the evolving strains lived under different environmental conditions and experienced different selection pressures, the new GEIs underwent various changes at different rates. GEIs of some strains, such as WSM1271, WSM2075, and WSM2073, maintained high conservation under weak selection pressures. GEIs of strains such as 7653R and MAFF303099, however, underwent frequent recombination events that created high levels of instability. In particular, GEIs of 7653R and MAFF303099 both encode mobility enzymes, such as integrase, that allow excision from the host chromosome. Nevertheless, only the original GEI of 7653R can excise itself spontaneously from the chromosome and form replicable plasmids. The GEI of MAFF303099 may have become immobilized because of failure to regain the origins of plasmid replication or the genes involved in mobilization [37].

Many transposase genes exist within GEIs of 7653R and MAFF303099. Except for several conserved but inactive genes, these genes were acquired from foreign species. The transposases encoded by foreign genes have retained high activity, indicating a continuous exchange of 7653R and MAFF303099 genetic information with other species. How rhizobial genomes are able to select the proper foreign genes while still maintaining structural stability and gene function despite the disruption remains unknown. Complex cellular programs associated with some bacterial traits, such as symbiosis, must exist to ensure adaption to the surrounding environment and to maintain competitiveness. A large body of research has confirmed this point. In one recent case, genes on a genomic island were reported to confer an adaptive advantage to specific stresses in marine Synechococcus [44]. For better survival and growth in various habitats, GEIs from MAFF303099 acquired some foreign nodulation genes by HGT during the genetic information exchange process, enabling functional symbiosis between MAFF303099 and a new host plant. Furthermore, the acquisition of foreign genetic elements is frequently accompanied by the loss of native genes. As to the argument that the lost genes are randomly selected or under special selection, increasing evidence inclines to the view 


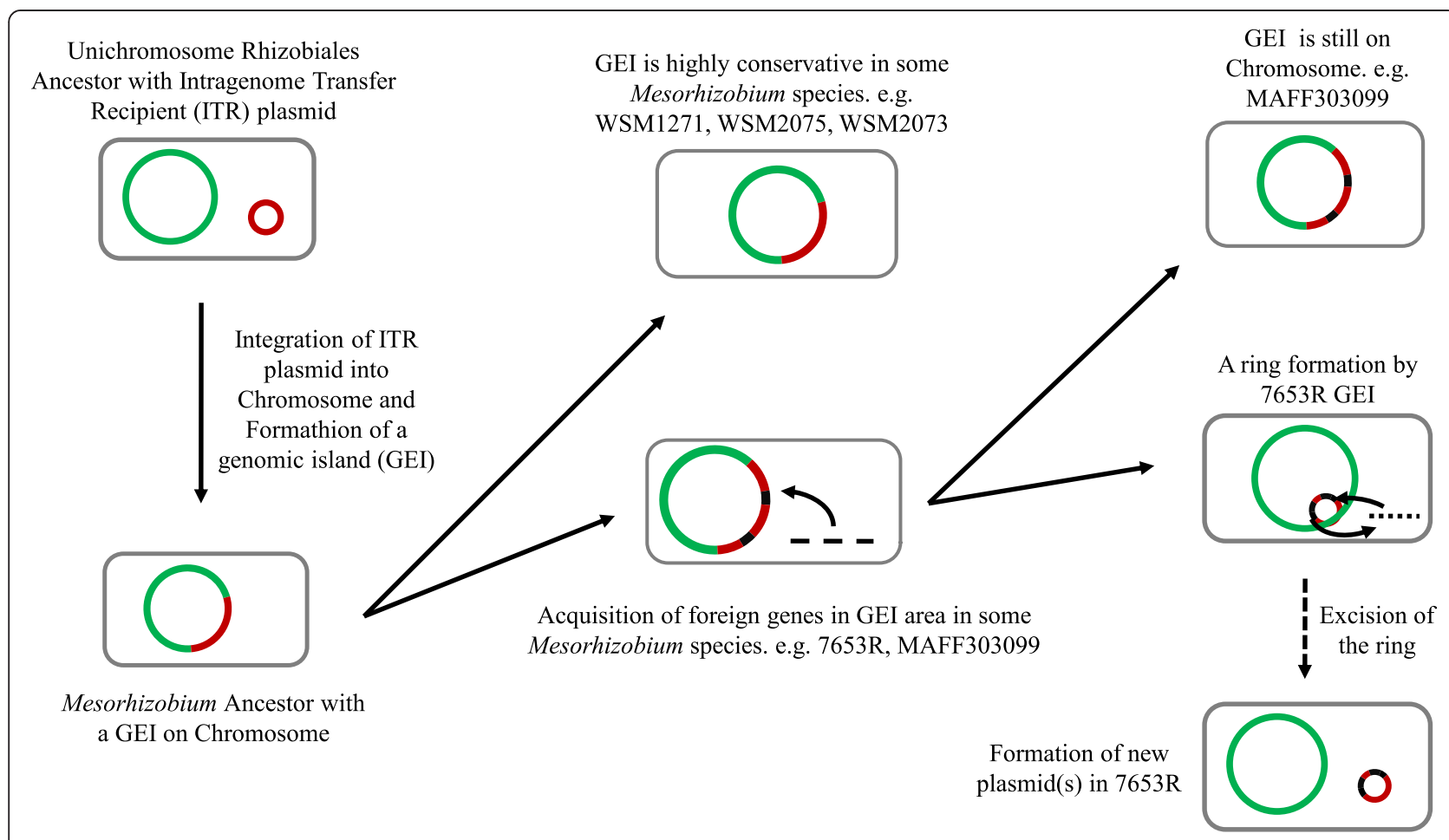

Figure 9 The contribution of genome dynamics to general Mesorhizobium evolution. Chromosomal, ITR plasmid, and foreign genes are shown in different colors.

that loss of functionality can be a selective advantage in some specific situations [45].

In Legionella pneumophila, a newly identified conjugation/type-IVA secretion system (trb/tra) composed of clusters of tra and trb genes (related to the Vir system and conjugal transformation) seems to be necessary for integrase-dependent excision and horizontal transfer of GEIs [46]. A similar system has been identified on the other four GEIs, excluding 7653R, with different sets of tra and $t r b$ genes scattered on them. The existence of the same set of tra and trb genes with high similarities in strains MAFF303099, WSM1271, WSM2075, WSM2073, and R7A [12] indicates that the ancestral ITR plasmid that integrated into chromosomes of ancestral Mesorhizobium strains contained a functional conjugation/ type-IVA secretion system. Plasmid pMhu7653Ra of 7653R, however, has only a few tra genes and no trb gene. Integrated mobile elements should theoretically be inactivated or lose genes related to plasmid mobilization or transfer, such as tra and trb. It is difficult to judge whether the IVA systems are inactive or if some of the key tra-trb genes have already been deleted from the GEIs of MAFF303099, WSM1271, WSM2075, and WSM2073. To determine what happened to the tratrb genes on the GEI of 7653R chromosome before excision, further bioinformatics analysis and experimental evidence are needed.

\section{Conclusions}

Whole-genome sequencing has proven valuable and critical for refining the phylogenetic positions of a series of rhizobial strains [47]. In this study, we sequenced, assembled, and annotated the M. huakuaii 7653R genome. We used this genome sequence to examine the phylogenetic position of MAFF303099, a strain whose phylogenetic position has been debated. These two strains share a large set of orthologs and, most importantly, a conserved chromosomal backbone and even larger perfectly conserved synteny blocks. Our ortholog and synteny analyses have firmly placed MAFF303099 as a strain of M. huakuii, as is 7653R.

Although 7653R and MAFF303099 are both strains of $M$. huakuii, they exhibit important differences in symbiotic phenotypes and thus belong to different symbiosis variants (also known as symbiovars) [48]. This placement is supported by our analysis of nodulation and fixation genes, which revealed notable differences in several nodulation genes, mostly related to NF generation. Such differences have a profound impact on host specificity. In a few rhizobium strains, mutations of some specific genes related to NFs and T3SS have been found to alter host specificity; additionally, the distribution of nodulation genes is reportedly related to requirements for effective symbiosis with some legume hosts [49-51]. Furthermore, our analysis of the three groups of signaling molecules 
revealed substantial differences between the two $M$. huakuii strains 7653R and MAFF303099 that were focused on the number and arrangement of genes responsible for synthesizing NFs and secretion systems T3SS, T4SS, and T6SS. In conjunction with NFs, these secretion systems may contribute to the establishment of differential host specificity.

Our results strongly suggest a common site-specific GEI localization mechanism in the ancestral Mesorhizobium chromosome, with the GEIs of the genus showing different degrees of variability after divergence from the mesorhizobial ancestor. A similar phenomenon has been observed in Bradyrhizobium japonicum strains. Various lines of evidence support past horizontal insertion of GEIs into the ancestral genome of B. japonicum USDA110, and comparative genomic hybridization profiles show that GEIs may be highly dynamic entities in $B$. japonicum genomes [52]. The ability of integrating mobile genetic elements to enlarge chromosomes may be due to the fact that Bradyrhizobium and Mesorhizobium species have very large chromosomes with few plasmids [43]. The recent completion of genome-sequencing projects for several Mesorhizobium species has enabled analysis of the global changes between them after the acquisition and integration of the ancestral ITR plasmid. An improved understanding of these variations should improve our understanding of how genome dynamics can contribute to bacterial evolution in general.

$7653 \mathrm{R}$ plasmids possess the same characteristics as the GEIs of the other four Mesorhizobium genomes. Additionally, homologs of nodulation and nitrogen-fixation genes on the other four GEIs are found on the two plasmids of 7653R. Moreover, it has been reported that GEIs can excise themselves spontaneously from the chromosome and form plasmids with the acquisition of functions for autonomous replication (e.g., rep $A B C$ genes) or can be transferred to other suitable recipients [53]. We therefore conclude that 7653R plasmids may have arisen by the excision of the original GEI from the 7653R chromosome.

\section{Methods}

\section{Bacterial strains and DNA preparation}

Mesorhizobium huakuii 7653R was cultured for 3 days at $28^{\circ} \mathrm{C}$ in trypticase-yeast extract medium. Cells of $7653 \mathrm{R}$ were harvested by centrifugation, with total DNA prepared using a Genomic DNA Mini Preparation kit.

\section{Sequencing and annotation}

For de novo sequencing of the 7653R genome, a combined strategy comprising Solexa sequencing on an Illumina GAIIx platform was carried out by BGI (Beijing Genomics Institute, Beijing, China). As a result, 367 contigs were generated with a 29-fold median coverage depth.
Sequence assembly was performed using SOAPdenovo [54], with PCR-based amplicon sequencing used for gap closure. Glimmer 3.0 [55], RNAmmer 1.2 [56], and tRNAscan-SE [57] were used respectively for de novo prediction of genes, rRNA genes, and tRNAs. Clusters of Orthologous Groups (COG) annotation was performed using RPS-BLAST against the CDD database [58], and Gene Ontology annotation was carried out with InterProScan V4 [59]. A bidirectional best hit approach $\left(E\right.$-value $<1 \times 10^{-5}$, identity $>30 \%$, coverage $>$ $70 \%$, and bit score $>60$ ) was used for KEGG [60] and SWISS-PROT [61] annotations.

\section{Genome comparisons}

The complete nucleotide sequences of strains MAFF30 3099, WSM1271, WSM2075, and WSM2073 were obtained from GenBank (accession numbers: M. huakuii bv. loti, NC_002678, NC_002679, and NC_002682; M. ciceri, NC_014923 and NC_014918; M. opportunistum, NC_015 675; M. australicum, NC_019973). The sequences were organized according to their chromosomal origins of replication for intuitive comparison. Sequences of three contigs from R7A were obtained from the JGI Genome Portal (Project ID: 404030). Genome sequence alignments were created using MUMmer, ACT, and Mauve software.

\section{Ortholog analysis}

The OrthoMCL [62] approach was adopted to construct gene families for all coding sequences in the five Mesorhizobium genomes. Quartets of orthologous proteins (quartops) in all pairwise genome comparisons were considered to constitute the 'core' genome. Proteins with no homologs in the other four Mesorhizobium genomes were defined as differential genes.

\section{Nucleotide sequence accession numbers}

Complete genome sequences of $M$. huakuii 7653R have been submitted to GenBank under the following assigned accession numbers: Mesorhizobium CP006581; Mesorhizobium_1 CP006582; Mesorhizobium_2 CP006583.

\section{Additional files}

\footnotetext{
Additional file 1: Tables S1, S4, S5, and S6. Table S1 The numbers and types of tRNAs in five mesorhizobial genome. Table S4 Similarities (\%) for nitrogen fixation genes of 7653R, R7A, WSM1271, and WSM2075 in comparison with those of MAFF303099. Table S5 Similarities (\%) of EPS biosynthesis genes of 7653R, WSM1271, WSM2075, and WSM2073 in comparison with those of MAFF303099. Table S6 Similarities (\%) of LPS biosynthesis genes of 7653R in comparison with those of MAFF303099, WSM1271, WSM2075, and WSM2073.
}

Additional file 2: Table S2. A list of 210 conserved genes in Mesorhizobium huakuii 7653R used for hierarchical clustering analysis.

Additional file 3: Table S3. $P$-values for $t$-test of significance. 
Additional file 4: Figures S1 to S5. Figure S1 Nodulation genes participating in NF synthesis. nodE and/or nodF from the 7653R chromosome, pMhu7653Rb, or both participate in synthesis of NFs. Figure S2 Lipopolysaccharide biosynthesis pathway in Mesorhizobium huakuii 7653R. Biosynthesis substrates and products and key enzymes of each step are indicated. Figure S3 ACT visualization of 7653R, R7A, and MAFF303099 chromosomes and plasmids. Genomic alignment of strains 7653R, R7A, and MAFF303099 was performed using ACT [38]. Red connections represent syntenic regions; blue ones represent inversions. The R7A genome with contigs in the order of contigs 1,2 , and 3 is at the top of the figure. The 7653R genome with replicons in the order of Chromosome, pMhu7653Ra and pMhu7653Rb is in the middle and the MAFF303099 genome is at the bottom in the order of Chromosome, pMLa, and pMLb. Figure S4 Genomic islands (GEIs) predicted for the four Mesorhizobium strains by IslandViewer. GEls are shown for MAFF303099 (A); WSM1271 (B); WSM2073 (C); and WSM2075 (D). Genomes in EMBL or GENBANK format are used. The green ellipse indicates the position of the GEl, which is the same as the symbiosis island on each chromosome. Figure S5 Comparison of codon usage among genomic islands (GEls) in Mesorhizobium. Codon usage patterns were compared between GEls and the remaining chromosomes. Lysine codon usages are not included because of the huge variability.

Additional file 5: Table S7. Proteins related to the type-III secretion system in Mesorhizobium huakuii 7653R and MAFF303099.

\section{Abbreviations}

CBG: Cyclic-ß-glucan; EPS: Exopolysaccharide; GEl: Genomic island; HGT: Horizontal gene transfer; ITR: Intragenomic translocation recipient; KPS: Capsular polysaccharide; LPS: Lipopolysaccharide; PID: Percentage identity; NF: Nodulation factor.

\section{Competing interests}

The authors declare that they have no competing interests.

\section{Authors' contributions}

$Y L, S W, H G, J P$, and FX designed the research. BH, SW, and BM performed the bioinformatics and statistical analyses, and $\mathrm{JL}, \mathrm{XZ}$, and CF carried out OrthoCluster and PROmer analyses. SW drafted the manuscript, and NC, BM, and $\mathrm{YL}$ were involved in critical revision. All authors read and approved the final manuscript.

\section{Acknowledgement}

This work was supported by funds from the National Basic Research Program of China (973 Program; 2010CB126502), the Research Fund for the Doctoral Program of Higher Education of China (20110146110012), the Fundamental Research Funds for the Central Universities (2009PY020 and 2010QC016), the National Natural Science Foundation of China (30970074 and 31100602), and the Natural Sciences and Engineering Research Council of Canada to NC. NC is also a Michael Smith Foundation for Health Research Scholar and a Canadian Institutes of Health Research New Investigator.

\section{Author details}

${ }^{1}$ State Key Laboratory of Agricultural Microbiology, Huazhong Agricultural University, Wuhan, Hubei, P. R. China. ${ }^{2}$ Center for Bioinformatics, School of Life Science and Technology, Huazhong Agricultural University, Wuhan, Hubei, P. R. China. ${ }^{3}$ Department of Molecular Biology and Biochemistry, Simon Fraser University, Burnaby, British Columbia, Canada.

Received: 15 November 2013 Accepted: 20 May 2014 Published: 6 June 2014

\section{References}

1. Masson-Boivin C, Giraud E, Perret X, Batut J: Establishing nitrogen-fixing symbiosis with legumes: how many rhizobium recipes? Trends Microbiol 2009, 17:458-466.

2. The current taxonomy of rhizobia. [http://www.rhizobia.co.nz/taxonomy/ rhizobia]

3. Degefu T, Wolde-Meskel E, Liu B, Cleenwerck I, Willems A, Frostegard A: Mesorhizobium shonense sp. nov., Mesorhizobium hawassense sp. nov. and
Mesorhizobium abyssinicae sp. nov. isolated from root nodules of different agroforestry legume trees. Int I Syst Evol Microbiol 2013, 63:1746-1753.

4. CHEN HK, SHU MK: Note on the root-nodule bacteria of Astragalus Sinicus L. Soil Sci 1944, 58:291-294.

5. Chen W, Li G, Qi Y, Wang E, Yuan HL, Li J: Rhizobium huakuii sp. nov. isolated from the root nodules of Astragalus sinicus. Int I Syst Bacteriol 1991, 41:275-280.

6. Jarvis B, Van Berkum P. Chen W, Nour S, Fernandez M, Cleyet-Marel J Gillis M: Transfer of Rhizobium loti, Rhizobium huakuii, Rhizobium ciceri, Rhizobium mediterraneum, and Rhizobium tianshanense to Mesorhizobium gen. nov. Int J Syst Bacteriol 1997, 47:895-898.

7. Cheng G, Li Y, Xie B, Yang C, Zhou J: Cloning and identification of IpsH, a novel gene playing a fundamental role in symbiotic nitrogen fixation of Mesorhizobium huakuii. Curr Microbiol 2007, 54:371-375.

8. Cheng GJ, Li YG, Zhou JC: Cloning and identification of opa22, a new gene involved in nodule formation by Mesorhizobium huakuii. FEMS Microbiol Lett 2006, 257:152-157.

9. Xie F, Cheng G, Xu H, Wang Z, Lei L, Li Y: Identification of a novel gene for biosynthesis of a bacteroid-specific electron carrier menaquinone. PLOS One 2011, 6:e28995.

10. Kaneko T, Nakamura Y, Sato S, Asamizu E, Kato T, Sasamoto S, Watanabe A Idesawa K, Ishikawa A, Kawashima K, Kimura T, Kishida Y, Kiyokawa C, Kohara M, Matsumoto M, Matsuno A, Mochizuki Y, Nakayama S, Nakazaki N, Shimpo S, Sugimoto M, Takeuchi C, Yamada M, Tabata S: Complete genome structure of the nitrogen-fixing symbiotic bacterium Mesorhizobium loti. DNA Res 2000, 7:331-338.

11. Turner SL, Zhang XX, Li FD, Young JP: What does a bacterial genome sequence represent? Mis-assignment of MAFF 303099 to the genospecies Mesorhizobium loti. Microbiology 2002, 148:3330-3331.

12. Sullivan JT, Trzebiatowski JR, Cruickshank RW, Gouzy J, Brown SD, Elliot RM Fleetwood DJ, McCallum NG, Rossbach U, Stuart GS, Weaver JE, Webby RJ, De Bruijn FJ, Ronson CW: Comparative sequence analysis of the symbiosis island of Mesorhizobium loti strain R7A. J Bacteriol 2002, 184:3086-3095.

13. Sullivan JT, Brown SD, Ronson CW: The NifA-RpoN Regulon of Mesorhizobium loti Strain R7A and Its Symbiotic Activation by a Novel Lacl/GalR-Family Regulator. PLoS One 2013, 8:e53762.

14. Hubber A, Vergunst AC, Sullivan JT, Hooykaas PJ, Ronson CW: Symbiotic phenotypes and translocated effector proteins of the Mesorhizobium loti strain R7A VirB/D4 type IV secretion system. Mol Microbiol 2004, 54:561-574

15. Hubber AM, Sullivan JT, Ronson CW: Symbiosis-induced cascade regulation of the Mesorhizobium loti R7A VirB/D4 type IV secretion system. Mol Plant Microbe Interact 2007, 20:255-261.

16. Estrella MJ, Muñoz S, Soto MJ, Ruiz O, Sanjuán J: Genetic diversity and host range of rhizobia nodulating Lotus tenuis in typical soils of the Salado River Basin (Argentina). Appl Environ Microbiol 2009, 75:1088-1098.

17. Zhang XX, Turner SL, Guo XW, Yang HJ, Debelle F, Yang GP, Denarie J, Young JP, Li FD: The common nodulation genes of Astragalus sinicus rhizobia are conserved despite chromosomal diversity. Appl Environ Microbiol 2000, 66:2988-2995.

18. Nanadasena K, Yates R, Tiwari R, O'Hara G, Howieson J, Ninawi M, Chertkov O, Detter C, Tapia R, Han S, Woyke T, Pitluck S, Nolan M, Land M, Liolios K, Pati A, Copeland A, Kyrpides NC, Ivanova N, Goodwin L, Meenakshi U, Reeve W: Complete genome sequence of Mesorhizobium ciceri bv. biserrulae type strain (WSM1271 ${ }^{\top}$ ). Stand Genomic Sci 2013, 9:1944-3277.

19. Nandasena KG, O'Hara GW, Tiwari RP, Willems A, Howieson JG: Mesorhizobium australicum sp. nov. and Mesorhizobium opportunistum sp. nov., isolated from Biserrula pelecinus L. in Australia. Int J Syst Evol Microbiol 2009, 59:2140-2147.

20. Reeve W, Nandasena K, Yates R, Tiwari R, O'Hara G, Ninawi M, Gu W, Goodwin L, Detter C, Tapia R, Han C, Copeland A, Liolios K, Chen A, Markowitz V, Pati A, Mavromatis K, Woyke T, Kyrpides N, Ivanova N, Howieson J: Complete genome sequence of Mesorhizobium australicum type strain (WSM2073 ${ }^{\mathrm{T}}$. Stand Genomic Sci 2013, 9:410-419.

21. Reeve WG, Nandasena K, Yates R, Tiwari R, O'Hara G, Ninawi M, Chertkov O, Goodwin L, Bruce D, Detter C, Tapia R, Han S, Woyke T, Pitluck S, Nolan M, Land M, Copeland A, Liolios K, Pati A, Mavromatis K, Markowitz V, Kyrpides $\mathrm{N}$, Ivanova N, Goodwin L, Meenakshi U, Howieson J: Complete genome sequence of Mesorhizobium opportunistum type strain WSM2075 ${ }^{\top}$. Stand Genomic Sci 2013, 9:1944-3277. 
22. Krzywinski M, Schein J, Birol I, Connors J, Gascoyne R, Horsman D, Jones SJ, Marra MA: Circos: an information aesthetic for comparative genomics. Genome Res 2009, 19:1639-1645.

23. Salama N, Guillemin K, McDaniel TK, Sherlock G, Tompkins L, Falkow S: A whole-genome microarray reveals genetic diversity among Helicobacter pylori strains. Proc Natl Acad Sci 2000, 97:14668-14673.

24. Darling AE, Mau B, Perna NT: progressiveMauve: multiple genome alignment with gene gain, loss and rearrangement. PLoS One 2010, 5:e11147.

25. Zeng $X$, Pei J, Vergara IA, Nesbitt $M$, Wang $K$, Chen N: OrthoCluster: a new tool for mining synteny blocks and applications in comparative genomics. In Proceedings of the 11th international conference on Extending database technology: Advances in database technology. Nantes, France: ACM; 2008:656-667.

26. Ng MP, Vergara IA, Frech C, Chen Q, Zeng X, Pei J, Chen N: OrthoClusterDB: an online platform for synteny blocks. BMC Bioinformatics 2009, 10:192

27. Xu Z, Hao B: CVTree update: a newly designed phylogenetic study platform using composition vectors and whole genomes. Nucleic Acids Res 2009, 37:W174-W178.

28. Yang S, Tang F, Gao M, Krishnan HB, Zhu H: R gene-controlled host specificity in the legume-rhizobia symbiosis. Proc Natl Acad Sci 2010, 107:18735-18740.

29. Kirzinger MW, Stavrinides J: Host specificity determinants as a genetic continuum. Trends Microbiol 2012, 20:88-93.

30. Fauvart M, Michiels J: Rhizobial secreted proteins as determinants of host specificity in the Rhizobium-legume symbiosis. FEMS Microbiol Lett 2008, 285:1-9.

31. Catoira R, Galera C, de Billy F, Penmetsa RV, Journet EP, Maillet F, Rosenberg C, Cook D, Gough C, Denarie J: Four genes of Medicago truncatula controlling components of a nod factor transduction pathway. Plant Cell 2000, 12:1647-1666.

32. Dilworth MJ, James EK, Sprent JI, Newton WE: Nitrogen-fixing Leguminous Symbioses. Springer; 2010

33. Janczarek M, Kutkowska J, Piersiak T, Skorupska A: Rhizobium leguminosarum bv. trifolii rosR is required for interaction with clover, biofilm formation and adaptation to the environment. BMC Microbiol 2010, 10:284.

34. Schmeisser C, Liesegang H, Krysciak D, Bakkou N, Le Quere A, Wollherr A, Heinemeyer I, Morgenstern B, Pommerening-Roser A, Flores M, Palacios R, Brenner S, Gottschalk G, Schmitz RA, Broughton WJ, Perret X, Strittmatter AW, Streit WR: Rhizobium sp. strain NGR234 possesses a remarkable number of secretion systems. Appl Environ Microbiol 2009, 75:4035-4045.

35. Sanchez C, lannino F, Deakin WJ, Ugalde RA, Lepek VC: Characterization of the Mesorhizobium loti MAFF303099 type-three protein secretion system. Mol Plant Microbe Interact 2009, 22:519-528.

36. Records AR: The type VI secretion system: a multipurpose delivery system with a phage-like machinery. Mol Plant Microbe Interact 2011 24:751-757.

37. Dobrindt U, Hochhut B, Hentschel U, Hacker J: Genomic islands in pathogenic and environmental microorganisms. Nat Rev Microbiol 2004, 2:414-424.

38. Carver TJ, Rutherford KM, Berriman M, Rajandream MA, Barrell BG, Parkhill J: ACT: the Artemis comparison tool. Bioinformatics 2005, 21:3422-3423.

39. Kurtz S, Phillippy A, Delcher AL, Smoot M, Shumway M, Antonescu C, Salzberg SL: Versatile and open software for comparing large genomes. Genome Biol 2004, 5:R12.

40. Langille MG, Brinkman FS: IslandViewer: an integrated interface for computational identification and visualization of genomic islands. Bioinformatics 2009, 25:664-665.

41. Antonenka U: Factors and Mechanisms of Mobility of the High Pathogenicity Island of Yersinia. 2007

42. Welch RA, Burland V, Plunkett G 3rd, Redford P, Roesch P, Rasko D, Buckles EL, Liou SR, Boutin A, Hackett J, Stroud D, Mayhew GF, Rose DJ, Zhou S, Schwartz DC, Perna NT, Mobley HL, Donnenberg MS, Blattner FR: Extensive mosaic structure revealed by the complete genome sequence of uropathogenic Escherichia coli. Proc Natl Acad Sci U S A 2002, 99:17020-17024.

43. Slater SC, Goldman BS, Goodner B, Setubal JC, Farrand SK, Nester EW, Burr TJ, Banta L, Dickerman AW, Paulsen I, Otten L, Suen G, Welch R, Almeida NF, Arnold F, Burton OT, Du Z, Ewing A, Godsy E, Heisel S, Houmiel KL, Jhaveri J,
Lu J, Miller NM, Norton S, Chen Q, Phoolcharoen W, Ohlin V, Ondrusek D, Pride N, et al: Genome sequences of three agrobacterium biovars help elucidate the evolution of multichromosome genomes in bacteria. J Bacteriol 2009, 191:2501-2511.

44. Fox GE, Wisotzkey JD, Jurtshuk P: How close is close: 16S rRNA sequence identity may not be sufficient to guarantee species identity. Int J Syst Bacteriol 1992, 42:166-170.

45. Maurelli AT, Fernandez RE, Bloch CA, Rode CK, Fasano A: "Black holes" and bacterial pathogenicity: a large genomic deletion that enhances the virulence of Shigella spp. and enteroinvasive Escherichia coli. Proc Natl Acad Sci U S A 1998, 95:3943-3948.

46. Glockner G, Albert-Weissenberger C, Weinmann E, Jacobi S, Schunder E, Steinert M, Hacker J, Heuner K: Identification and characterization of a new conjugation/type IVA secretion system (trb/tra) of Legionella pneumophila Corby localized on two mobile genomic islands. Int J Med Microbiol 2008, 298:411-428.

47. Schuldes J, Rodriguez Orbegoso M, Schmeisser C, Krishnan HB, Daniel R, Streit WR: Complete genome sequence of the broad-host-range strain Sinorhizobium fredii USDA257. J Bacterio/ 2012, 194:4483.

48. Rogel MA, Ormeño-Orrillo E, Martinez Romero E: Symbiovars in rhizobia reflect bacterial adaptation to legumes. Syst Appl Microbio/ 2011, 34:96-104.

49. Dai WJ, Zeng Y, Xie ZP, Staehelin C: Symbiosis-promoting and deleterious effects of NopT, a novel type 3 effector of Rhizobium sp. strain NGR234. J Bacteriol 2008, 190:5101-5110.

50. Moulin L, Bena G, Boivin-Masson C, Stepkowski T: Phylogenetic analyses of symbiotic nodulation genes support vertical and lateral gene co-transfer within the Bradyrhizobium genus. Mol Phylogenet Evol 2004, 30:720-732.

51. Schechter LM, Guenther J, Olcay EA, Jang S, Krishnan HB: Translocation of NopP by Sinorhizobium fredii USDA257 into Vigna unguiculata root nodules. Appl Environ Microbiol 2010, 76:3758-3761.

52. Itakura M, Saeki K, Omori H, Yokoyama T, Kaneko T, Tabata S, Ohwada T, Tajima S, Uchiumi T, Honnma K, Fujita K, Iwata H, Saeki Y, Hara Y, Ikeda S, Eda S, Mitsui H, Minamisawa K: Genomic comparison of Bradyrhizobium japonicum strains with different symbiotic nitrogen-fixing capabilities and other Bradyrhizobiaceae members. ISME J 2009, 3:326-339.

53. Benedek O, Schubert S: Mobility of the Yersinia High-Pathogenicity Island (HPI): transfer mechanisms of pathogenicity islands (PAIS) revisited (a review). Acta Microbiol Immunol Hung 2007, 54:89-105.

54. Luo R, Liu B, Xie Y, Li Z, Huang W, Yuan J, He G, Chen Y, Pan Q, Liu Y, Tang J, Wu G, Zhang H, Shi Y, Liu Y, Yu C, Wang B, Lu Y, Han C, Cheung DW, Yiu SM, Peng S, Xiaoqian Z, Liu G, Liao X, Li Y, Yang H, Wang J, Lam TW, Wang J: SOAPdenovo2: an empirically improved memory-efficient short-read de novo assembler. Gigascience 2012, 1:18.

55. Delcher AL, Harmon D, Kasif S, White O, Salzberg SL: Improved microbial gene identification with GLIMMER. Nucleic Acids Res 1999, 27:4636-4641.

56. Lagesen $\mathrm{K}$, Hallin $\mathrm{P}$, Rodland EA, Staerfeldt HH, Rognes T, Ussery DW: RNAmmer: consistent and rapid annotation of ribosomal RNA genes. Nucleic Acids Res 2007, 35:3100-3108.

57. Lowe TM, Eddy SR: tRNAscan-SE: a program for improved detection of transfer RNA genes in genomic sequence. Nucleic Acids Res 1997, 25:0955-0964

58. Marchler-Bauer A, Zheng C, Chitsaz F, Derbyshire MK, Geer LY, Geer RC, Gonzales NR, Gwadz M, Hurwitz DI, Lanczycki CJ, Lu F, Lu S, Marchler GH, Song JS, Thanki N, Yamashita RA, Zhang D, Bryant SH: CDD: conserved domains and protein three-dimensional structure. Nucleic Acids Res 2013, 41:D348-352.

59. Zdobnov EM, Apweiler R: InterProScan-an integration platform for the signature-recognition methods in InterPro. Bioinformatics 2001, 17:847-848

60. Kanehisa M, Goto S: KEGG: kyoto encyclopedia of genes and genomes. Nucleic Acids Res 2000, 28:27-30.

61. Bairoch A, Apweiler R: The SWISS-PROT protein sequence database and its supplement TrEMBL in 2000. Nucleic Acids Res 2000, 28:45-48,

62. Li L, Stoeckert CJ Jr, Roos DS: OrthoMCL: identification of ortholog groups for eukaryotic genomes. Genome Res 2003, 13:2178-2189.

doi:10.1186/1471-2164-15-440

Cite this article as: Wang et al:: Whole-genome sequencing of Mesorhizobium huakuii 7653R provides molecular insights into host specificity and symbiosis island dynamics. BMC Genomics 2014 15:440. 